\title{
PERFORMANCE OF LINEAR SOLVERS APPLIED TO THE SOLUTION OF THE ELECTROMAGNETIC FORWARD PROBLEM IN THE FREQUENCY DOMAIN
}

Emanuelle Machado* (emanuellesilva@on.br), Sergio Fontes (sergio@on.br)* e Williams Lima (williamslima@on.br)*

*Observatório Nacional, RJ-Brasil

Copyright 2021, SBGf - Sociedade Brasileira de Geofísica.

This paper was prepared for presentation during the $17^{\text {th }}$ International Congress of the Brazilian Geophysical Society held in Rio de Janeiro, Brazil, $16-19$ August 2021.

Contents of this paper were reviewed by the Technical Committee of the $17^{\text {th }}$ International Congress of the Brazilian Geophysical Society and do not necessarily represent any position of the SBGf, its officers or members. Electronic reproduction or storage of any part of this paper for commercial purposes without the written consent of the Brazilian Geophysical Society is prohibited.

\begin{abstract}
Magnetotellurics (MT) and mCSEM are two very popular and important geophysical exploration methods. They both are based on the solution of Maxwell's equations in conductive media at low frequency regimes. Solving the Maxwell's equations in a general three-dimensional, non-homogenous, isotropic or anisotropic medium requires a numerical approach among which finite differences (FD) and finite elements (FE) are the most frequently employed. Both FD and FE methods reduce Maxwell's equations to an algebraic linear system of equations. It is very important then, to have very efficient linear solvers specially tailored for use in MT and mCSEM applications. There are basically two different groups of linear solvers more frequently applied in these applications: iterative and direct methods. Iterative methods are very efficient and are routinely used in many numerical codes. On the other hand, in some situations, iteratives methods may not be the most efficient and direct methods can become a sound alternative. It can be the case, for example, for mCSEM modeling and inversion when the number of sources becomes very big. In this work we show some preliminary results of a research that we are carrying out in order to assess the viability of the use of direct methods for the solution of large systems of linear algebraic equations resulting from the discretization of Maxwell's equations by finite differences method for modeling MT and mCSEM data. At the current stage of our investigations, we have solved the linear systems using two iterative algorithms, QMR and BiCG. We report in this work the performance of both algorithms in terms of runtime, accuracy and required number of iterations necessary for achieving a given solution accuracy. We performed MT numerical modeling experiments using the very known 3D conductivity model Dublin Test Model 1 (DTM1) for a number of different MT periods. Our findings indicate that BiCG far outperforms QMR in both iterations count and runtime for all the cases considered. In our next steps we are going to include more iterative algorithms, experiment with different pre-conditioners and add a direct solver to our base 3D code. We believe that our results will help the geophysical community, working with modeling and inversion of EM data, to decide upon the most appropriate solver for each particular scenario. Moreover, because the solution of systems of linear equations are very common in many other applications, our research will have the potential to have a much broader contribution outside EM methods and even other than geophysics research fields.
\end{abstract}

